\title{
Hydrogen in tungsten trioxide by membrane photoemission and density functional theory modeling
}

\author{
Emanuel Billeter $\odot,{ }^{1,2}$ Andrea Sterzi, ${ }^{1}$ Olga Sambalova, ${ }^{1,2}$ René Wick-Joliat $\odot,{ }^{2}$ Cesare Grazioli $\odot,{ }^{3}$ Marcello Coreno $\odot,{ }^{4}$ \\ Yongqiang Cheng, ${ }^{5}$ Anibal J. Ramirez-Cuesta, ${ }^{5}$ and Andreas Borgschulte $\odot^{1,2, *}$ \\ ${ }^{1}$ Laboratory for Advanced Analytical Technologies, Empa - Swiss Federal Laboratories for Materials Science and Technology, \\ Überlandstrasse 129, 8600 Dübendorf, Switzerland \\ ${ }^{2}$ Department of Chemistry, University of Zürich, Winterthurerstrasse 190, 8057 Zürich, Switzerland \\ ${ }^{3}$ IOM-CNR, Laboratorio TASC, Basovizza SS-14, km 163.5, 34149 Trieste, Italy \\ ${ }^{4}$ ISM-CNR, Istituto di Struttura della Materia, LD2 Unit, 34149 Trieste, Italy \\ ${ }^{5}$ Neutron Scattering Division, Oak Ridge National Laboratory, Oak Ridge, Tennessee 37831, USA
}

(Received 24 June 2020; revised 9 April 2021; accepted 20 April 2021; published 10 May 2021)

\begin{abstract}
The measurement of hydrogen-induced changes in the electronic structure of transition metal oxides by x-ray photoelectron spectroscopy is a challenging endeavor, since no photoelectron can be unambiguously assigned to hydrogen. The $\mathrm{H}$-induced electronic structure changes in tungsten trioxide have been known for more than 100 years but are still controversially debated. The controversy stems from the difficulty in disentangling effects due to hydrogenation from the effects of oxygen deficiencies. Using a membrane approach to x-ray photoelectron spectroscopy, in combination with tunable synchrotron radiation, we measure simultaneously core levels and the valence band up to a hydrogen pressure of 1000 mbar. Upon hydrogenation, the intensities of the $\mathrm{W}^{5+}$ core level and a state close to the Fermi level increase following the pressure-composition isotherm curve of bulk $\mathrm{H}_{x} \mathrm{WO}_{3}$. Combining experimental data and density functional theory, the description of the hydrogen-induced coloration by a proton polaron model is corroborated. Although hydrogen is the origin of the electronic structure changes near the Fermi edge, the valence band edge is now dominated by tungsten orbitals instead of oxygen as is the case for the pristine oxide, having wider implications for its use as a (photoelectrochemical) catalyst.
\end{abstract}

DOI: 10.1103/PhysRevB.103.205304

\section{INTRODUCTION}

Hydrogen is a ubiquitous element in the environment. The element plays a key role in biology, chemistry, and physics: It is involved in numerous chemical reactions, from photosynthesis to the combustion of its products, and plays an essential role in corrosion processes. The fast diffusion of hydrogen in most materials, including nonorganic matter such as oxides, makes hydrogen an omnipresent impurity [1]. Due to its polyvalent chemical character, hydrogen in matter may be present as a proton, as a neutral atom, or as an anion. As a consequence of its atomic number, hydrogen has only one electron. Particularly, this property is a challenge for many analytical tools based on the interaction with electrons: Core-level spectroscopies such as X-ray photoelectron spectroscopy (XPS) cannot be used as a quantitative method for hydrogen, because a photoemitted electron cannot be assigned to a hydrogen atom without any doubt, $\mathrm{H}^{+}$(e.g., $\mathrm{OH}$ ) has formally no electrons, $\mathrm{H}^{-}$has two, and in covalent bonds the $\mathrm{H}$ electron has a high probability density between the binding atoms; that is, the location of hydrogen electrons depends on the electronic structure of the material. The electronic structure, though, is the key to understanding the physical and chemical properties of matter, and photoemission spectroscopy is the standard experimental method to unravel it.

*andreas.borgschulte@empa.ch
Obviously, materials in which the electronic structure is decisively influenced by hydrogen are particularly difficult to analyze. The archetypal example of this materials class is hydrogen-intercalated tungsten trioxide, $\mathrm{H}_{x} \mathrm{WO}_{3}$. Already in the nineteenth century, Berzelius noticed a color change when hydrogen gas was passed over tungsten trioxide [2]. Similar reversible optical and electrical changes are observed upon electrochemical insertion of alkali metals into $\mathrm{WO}_{3}$ [3]. The discovery of the electrochromic properties of thin $\mathrm{WO}_{3}$ films led to the development of a number of applications such as smart windows, displays, and variable mirrors [4,5]. As the above-discussed constraints for hydrogen do not apply here, the electronic structure of alkali-metal-intercalated tungsten bronzes, e.g., $\mathrm{Na}_{x} \mathrm{WO}_{3}$, are well characterized [6,7]. With the help of electronic structure modeling [8,9], there is consensus about the underlying physical phenomena of the chemical modification induced by alkali-metal intercalation [10]. In short, the electron of the alkali metal forms a new state in the band gap (conducting phase), but the phase initially remains insulating due to Coulomb interactions. In $\mathrm{Na}_{x} \mathrm{WO}_{3}$, a metalinsulator transition occurs at $x=0.24$; similar behavior is found for the other alkali-metal-intercalated tungsten bronzes $[9,11]$. Although intuitively similar, the changes taking place during hydrogen intercalation are different and controversially debated. Simplified, there are two models: Hydrogen is intercalated into $\mathrm{WO}_{3}$ and remains there, most likely as hydroxide; the corresponding electron affects the valence band in a similar way to the ones originating from alkali metals $[8,12]$. 
The second model proposes that hydrogen can form water with oxygen from $\mathrm{WO}_{3}$ and leave the now substoichiometric crystal [13]. The corresponding oxygen vacancies are filled with electrons. The loss of oxygen from the lattice induces distortions, leading to the localization of the electrons, that are described by an electron polaron model [14]. Summarizing, the origin of the controversy stems from the inability to detect and characterize hydrogen in the oxide.

In contrast to the electronic structure, the crystal structures of hydrogen and alkaline-metal-intercalated $\mathrm{WO}_{3}$ are well studied. Most alkaline metal bronzes have a perovskite structure, where the alkaline metal occupies the central position [3]. The structure of hydrogen-intercalated $\mathrm{WO}_{3}$ was determined by x-ray diffraction (XRD) and neutron diffraction on deuterium analogs $[15,16]$. It revealed a distorted cubic structure, where the hydrogen occupies a position $1.1 \AA$ from the oxygen along the diagonal through the central position. This was supported by density functional theory (DFT) calculations on cubic $\mathrm{WO}_{3}$ and $\mathrm{HWO}_{3}$ finding the minimum energy position of hydrogen in the $\mathrm{WO}_{3}$ lattice at a distance of $1.03 \AA$ from the oxygen atoms [8]. Electronic structures have been calculated for hydrogen and alkali-metal intercalation explaining the observed changes at high intercalant concentration $[8,17]$.

Experimentally, the intercalation process can be performed in two different ways. Electrochemical hydrogen insertion is relatively facile, but the electrochemical surface changes due to the aqueous environment hinder photoemission measurements. Gaseous hydrogen intercalation into $\mathrm{WO}_{3}$ does not change the surface but is feasible only at UHV-incompatible pressures and in the presence of dissociatively active sites [18], limiting photoemission experiments to the study of postmortem samples.

In this paper, we present in situ photoemission data obtained by the membrane photoemission approach [19]: We employ a sample holder that enables varying the hydrogen pressure up to 1 bar while keeping the tungsten oxide thin film under UHV conditions necessary for photoelectron spectroscopy experiments [19] (see Fig. 1). This allows us to measure the pressure-composition isotherm of $\mathrm{WO}_{3}$ by photoelectron spectroscopy. Synchrotron light enables probing of oxygen and tungsten core levels and the valence band states as a function of hydrogen content. The operando approach facilitates interpretation, as effects from unavoidable substoichiometries of the sample and other experimental uncertainties can be separated from the effects expected from hydrogen intercalation. Furthermore, we compare the photoemission results with DFT calculations, supporting the interpretation of experimental results in light of a polaron model.

\section{EXPERIMENT}

\section{A. Sample preparation}

$\mathrm{WO}_{3}$ was deposited on palladium by electrodeposition from a $\mathrm{H}_{2} \mathrm{WO}_{4}$ solution. The $\mathrm{H}_{2} \mathrm{WO}_{4}$ solution was prepared in a round-bottom flask by suspending $0.92 \mathrm{~g}$ tungsten powder (99.9\%; Sigma-Aldrich) in $7.5 \mathrm{ml}$ water. The suspension was heated to $60^{\circ} \mathrm{C}$, and $3.5 \mathrm{ml} \mathrm{H}_{2} \mathrm{O}_{2}$ (30\%; Merck) were

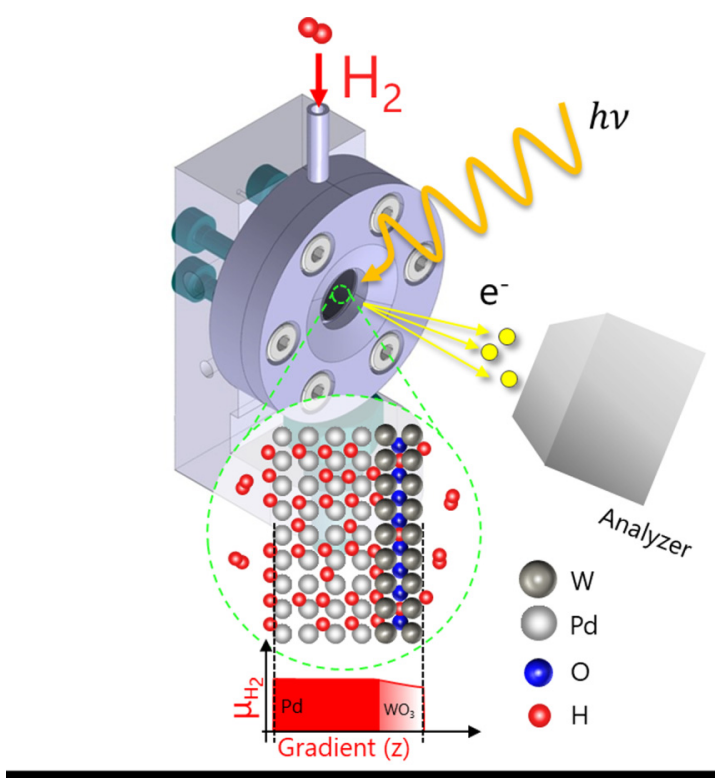

FIG. 1. Sketch of the membrane XPS sample holder and the experimental setup. The enlarged area shows the hydrogen permeation and the hydrogen chemical potential through the membrane. At steady state, the chemical potential on the feed side of the membrane is nearly equal to that on the vacuum side allowing the measurement of hydrogen concentrations in the material corresponding to atmospheric pressures by surface science techniques $[19,20]$.

added. Tungsten was oxidized to tungstic acid accompanied by vigorous gas formation $\left(\mathrm{H}_{2}\right)$, and after 2 min a colorless, clear solution was obtained. A platinum wire was added to decompose the excess of $\mathrm{H}_{2} \mathrm{O}_{2}$ until no more oxygen bubbles evolved, which took $8 \mathrm{~h}$ at $60^{\circ} \mathrm{C}$. A final tungstic acid concentration of $50 \mathrm{mM}$ was obtained by adding $50 \mathrm{ml}$ of isopropyl alcohol and filling up with water to a total volume of $100 \mathrm{ml}$. Electrodeposition was performed in a two-electrode setup. A $2 \times 2$-cm foil of Pd with $47 \mu \mathrm{m}$ thickness (purity 99.95\%; Goodfellow) with its back side covered with Kapton tape served as working electrode, and a Pt wire served as counterelectrode. Since the $\mathrm{WO}_{3}$ deposition on Pd competes with $\mathrm{H}_{2}$ formation, relatively high current densities of approximately $-2 \mathrm{~mA} / \mathrm{cm}^{2}$ were applied in chronopotentiometry mode for $10 \mathrm{~min}$, which resulted in an amorphous blue film of approximately $30 \mathrm{~nm}$ thickness, as determined by XRD and energy dispersive $\mathrm{x}$-ray (EDX) analysis, respectively. The sample was then dried in air on a hot plate at $200^{\circ} \mathrm{C}$ for 10 min.

\section{B. Membrane sample holder}

The high-pressure XPS study reported here is based on the Pd membrane approach that we previously presented [20]. The photoelectron spectroscopy measurements were carried out at the GasPhase beamline of the Elettra synchrotron light source in Trieste, Italy. The beamline is equipped with a dedicated differential pumping system [21]. The combination of a high-resolution monochromator with the high transmission of the electron analyzer allowed us to collect spectra with a minimal energy resolution of $50 \mathrm{meV}$ [22]. 
Briefly, the idea behind the embedded membrane approach is the following. The sample holder sketched in Fig. 1 consists of a hydrogen-permeable Pd membrane which is exposed on one side to high hydrogen pressure (up to 1 bar) and on the other side to UHV $\left(10^{-8}\right.$ mbar). Hydrogen is fluxed on the high-pressure side of the membrane, and following permeation, it reaches the surface exposed to vacuum in the form of atomic hydrogen. The tungsten oxide film, which is deposited on the analyzed side of the membrane, is thereby hydrogenated. Since the hydrogen permeation in the palladium membrane is much faster than in tungsten oxide, the oxide layer blocks hydrogen permeation. This creates a constant hydrogen concentration, and therefore a constant chemical potential, in the palladium membrane [19]. It is thereby possible to control the chemical potential of hydrogen on the UHV side of the membrane by the applied hydrogen pressure (see Fig. 1). The validity of the assumption of the equality of chemical potentials is experimentally evidenced by the permeation kinetics: The analysis gives surface-limited permeation (see Appendix, Fig. 9).

Photoemission measurements require this system to be in a pressure-temperature equilibrium state. In order to realize that, both the gas pressure and the membrane temperature were adjusted. The membrane was heated up to $160^{\circ} \mathrm{C}$ by means of a heating filament, and the hydrogen flux was controlled through a valve and a pressure gauge. Pressuredependent spectra were obtained as follows. Hydrogen was constantly fluxed inside the sample holder; an equilibrium condition is reached when the amount of absorbed gas compensates the desorption process which occurs on the UHV side of the membrane [20]. When this steady state has been reached, the pressures on the two sides of the membrane are stable, and the measurement can be performed. The hydrogen amount inside the experimental chamber and hence the desorption process were monitored by means of a quadrupole residual gas analyzer (see Appendix, Fig. 9).

\section{Photoelectron spectroscopy}

In situ synchrotron measurements were recorded using $700 \mathrm{eV}$ photon energy for the survey spectra and $104 \mathrm{eV}$ for the simultaneous detection of tungsten $4 f$ core levels and the valence band. During hydrogenation experiments, the photoemission spectra were recorded continuously in singlesweep mode, with each spectrum taking approximately $120 \mathrm{~s}$. The applied hydrogen pressure was measured by an external pressure gauge. As soon as the partial hydrogen pressure in the chamber was constant, the external hydrogen pressure was increased. This led to approximately ten spectra being recorded for each external hydrogen pressure. These spectra were averaged for the subsequent data evaluation to improve signal-to-noise ratios. Data analysis was performed using the CASAXPS software, employing the GL(30) line shape, Shirley background subtraction, and a 2.1-eV spin orbit splitting for both $\mathrm{W}^{5+}$ and $\mathrm{W}^{6+}$ doublets [23]. The $\mathrm{W} 5 p_{3 / 2}$ core level is not fitted in the spectra recorded at $104 \mathrm{eV}$, due to its small photoionization cross section at this photon energy [24]. The binding-energy calibration was performed on the gold-coated sample holder, setting the $\mathrm{Au} 4 f_{7 / 2}$ peak to $84.0 \mathrm{eV}$. Due to small photon energy instabilities and work function changes during hydrogenation, all spectra were subsequently aligned by shifting the $\mathrm{W}^{6+} 4 f_{7 / 2}$ peak to $35.8 \mathrm{eV}$. These shifts are on the order of $0.2-0.3 \mathrm{eV}$.

Ex situ measurements were performed using a PHI Quantes spectrometer (ULVAC-PHI) equipped with a monochromatic $\mathrm{Al} \mathrm{K} \alpha(1486.6 \mathrm{eV}) \mathrm{x}$-ray source. Charge neutralization was accomplished by a dual-beam charge neutralization system, employing low-energy electron and argon ion beams. (Detailed acquisition parameters are given in the Appendix, Table I.)

\section{Electronic structure calculation}

Calculation of electronic structure by density functional theory (DFT) and hybrid DFT was performed using the Vienna ab initio simulation package (VASP) [25-28]. The calculation used the projector augmented-wave (PAW) method [29,30] to describe the effects of core electrons, and Perdew-Burke-Ernzerhof (PBE) [31] implementation of the generalized gradient approximation (GGA) for the exchangecorrelation functional. The energy cutoff was $600 \mathrm{eV}$ for the plane-wave basis of the valence electrons. The electronic structure was calculated on a $15 \times 15 \times 15 \Gamma$-centered mesh for $\mathrm{WO}_{3}$ (unit cell), and a $4 \times 4 \times 4 \Gamma$-centered mesh for $\mathrm{H}_{x} \mathrm{WO}_{3}(2 \times 2 \times 2$ supercell $)$. The total energy tolerance for electronic energy minimization was $10^{-6} \mathrm{eV}$, and for structure optimization it was $10^{-5} \mathrm{eV}$. The maximum interatomic force after relaxation was below $0.01 \mathrm{eV} / \AA$. After structural optimization, hybrid-DFT calculation of the band structure was performed using the Heyd-Scuseria-Ernzerhof HSE06 hybrid functional $[32,33]$ with a mixing parameter of $25 \%$ and a screening parameter of $0.2 \mathrm{AA}^{-1}$.

Hydrogen-free $\mathrm{WO}_{3}$ crystallizes in the monoclinic structure with space group $P 2_{1} / n$ with $a=7.304 \AA, b=7.536$ $\AA, c=7.691 \AA$, and $\beta=90.85^{\circ}$ [34]. Other polymorphs exist depending on temperature [35]. However, intercalation of hydrogen leads to the formation of cubic structures of $\mathrm{H}_{x} \mathrm{WO}_{3}$ for $x=0.5$ [16]. We thus simplified the calculations using the cubic $\mathrm{ReO}_{3}$ structure where the metal is surrounded by six oxygen atoms in an octahedron (see Fig. 2) for all compositions including hydrogen-free $\mathrm{WO}_{3}$. With this constraint, the calculated electronic structure is that of an artificial structure with slight deviations from reality. A simple quality parameter is the optical gap $[17,36]$. The calculated direct band gap of simple cubic $\mathrm{WO}_{3}$ with around $2.3 \mathrm{eV}$ matches the experimental values of $2.6-3.3 \mathrm{eV}$ for the direct band gap [13,36,37]. This difference is due to the general underestimation of the optical gap by GGA and weighted density approximation (WDA) methods [38], with $\mathrm{WO}_{3}$ being particularly notorious [39]. Furthermore, calculations of the gap of the simple cubic phase yield smaller gaps than the ones calculated for the monoclinic phase of bulk tungsten trioxide $[8,17,40]$. Values vary from $0.69 \mathrm{eV}$ [revised PBE (RPBE)] and $2.25 \mathrm{eV}$ (PBE8) for the simple cubic system to $1.3 \mathrm{eV}$ (RPBE) and $3.68 \mathrm{eV}$ (PBE8) for the monoclinic system, with the functional indicated in brackets [39]. However, the simplicity of a cubic system allows the modeling of the partially hydrogenated bronzes $\mathrm{H}_{x} \mathrm{WO}_{3}$ with $x=0,0.25$, 0.75 , and 1.0. For $x=0.25$, two hydrogens with antiparallel spin configuration are placed in a simple cubic eightfold unit 
TABLE I. Acquisition parameters for XPS and synchrotron photoelectron spectroscopy.

\begin{tabular}{lcccc}
\hline \hline & Energy range (eV) & Pass energy (eV) & Energy step size (meV) & Total acquisition time per point (s) \\
\hline Ex situ XPS survey & $1100-0$ & 112 & 100 & 0.4 \\
Ex situ XPS narrow scan & variable & 55 & 50 & 1.2 \\
In situ XPS survey & $550-0$ & 20 & 200 & 0.6 \\
In situ XPS narrow scan & variable & 20 & 50 & 0.1 \\
\hline \hline
\end{tabular}

cell of $\mathrm{WO}_{3}$ (see Fig. 2 and the Appendix, Fig. 10). Nine different hydrogen constellations were calculated, with the two hydrogen atoms placed in the unit cells along the 100, 110 , and 111 directions, respectively. The difference between these constellations turned out to be negligible (Appendix, Fig. 10). The hydrogen and oxygen positions were released in the subunit to find the $\mathrm{H}-\mathrm{O}$ position with minimum total energy. We found an optimum for $d_{\mathrm{O}-\mathrm{H}}=1.03 \AA$, in good agreement with the literature [8]. In addition, the oxygen tetrahedrons were distorted (Fig. 2). Similar calculations were performed for $\mathrm{H}_{0.75} \mathrm{WO}_{3}$ using six hydrogens per eightfold unit cell. For better comparison, the calculated total density of electron states is broadened by $0.5 \mathrm{eV}$ to match the experimental resolution of photoemission spectroscopy (see Fig. 5).

\section{RESULTS}

For characterization, XPS measurements employing Al K $\alpha$ radiation were performed on the tungsten-oxide-coated palladium membrane pre- and posthydrogenation (see Appendix, Fig. 7). The results are in perfect agreement with the literature; that is, we find mainly $\mathrm{W}^{6+}$ and some $\mathrm{W}^{5+}$ as extracted by peak fitting of the $\mathrm{W} 4 f$ states [41], which is typical for amorphous $\mathrm{WO}_{3}$ thin films [42]. There is no significant difference between the data taken ex situ before and after hydrogen exposure. The survey spectra show less carbon contamination and the presence of $\mathrm{Pd} 3 d$ peaks after hydrogenation, the latter are attributed to inadvertent removal of the thin $\mathrm{WO}_{3}$ layer

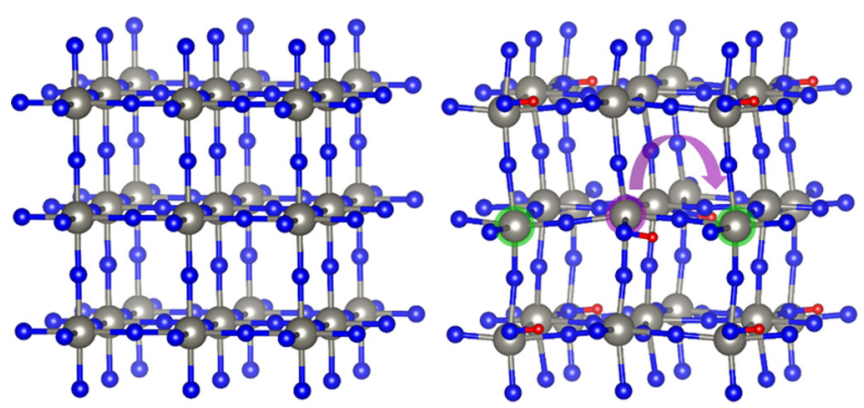

FIG. 2. Illustration of the cubic crystal structure of $\mathrm{WO}_{3}$ (left) and of one polymorph of $\mathrm{H}_{0.25} \mathrm{WO}_{3}$ (right) as used for modeling. Note the distortion of the oxygen atoms (blue) induced by hydrogen (red) amounts as little as $x=0.25$. This distortion is local: As an example, two tungsten atoms (gray) exclusively surrounded by oxygen atoms are highlighted with a green corona, and one tungsten atom surrounded by five oxygens and one $\mathrm{OH}$ group is highlighted with a purple corona. In the local picture, the latter atoms are $\mathrm{W}^{5+}$, and the ones surrounded solely by oxygen are $\mathrm{W}^{6+}$. Moving an electron and hydrogen (arrow) requires structural rearrangements. during mounting or dismounting from the membrane sample holder.

The same procedure was applied in situ directly before and after hydrogen exposure (Appendix, Fig. 8). After hydrogen exposure the ratio $\mathrm{W}^{5+} / \mathrm{W}^{6+}$ increases. The intensity of the higher-binding-energy shoulder of the $\mathrm{O} 1 s$ peak, indicative of $\mathrm{OH}$ and/or carbonate species [42], increases in parallel (Appendix, Fig. 8). These changes were followed in situ at various hydrogen pressures applied to the membrane. We observed continuous variations of core levels (Fig. 3) as well as the valence band [Fig. 5(b)] indicative of hydrogen intercalation into $\mathrm{WO}_{3}$. However, in addition to the effects assigned to hydrogen exposure, we found a higher $\mathrm{W}^{5+} / \mathrm{W}^{6+}$ ratio derived from in situ measurements at $700 \mathrm{eV}$ than the one derived $e x$ situ at $1486.6 \mathrm{eV}$ photon energy, both measured on the sample before hydrogen exposure. This effect is attributed to the prolonged UHV exposure necessary to align the experimental setup. During alignment and optimization of the acquisition parameters, the membrane was exposed to UV radiation of $104 \mathrm{eV}$ photon energy. It is well known that UV radiation induces the reduction of $\mathrm{WO}_{3}$, leading to larger fractions of $\mathrm{W}^{5+}$ [43]. This effect is a major detriment of photoelectron spectroscopy applied to such systems in general.

The in situ hydrogenation opens the possibility to disentangle the effects from radiation damage and hydrogen exposure by following the evolution of the spectra with and without hydrogen. In Fig. 3, the $\mathrm{WO}_{3}$ thin film was exposed to

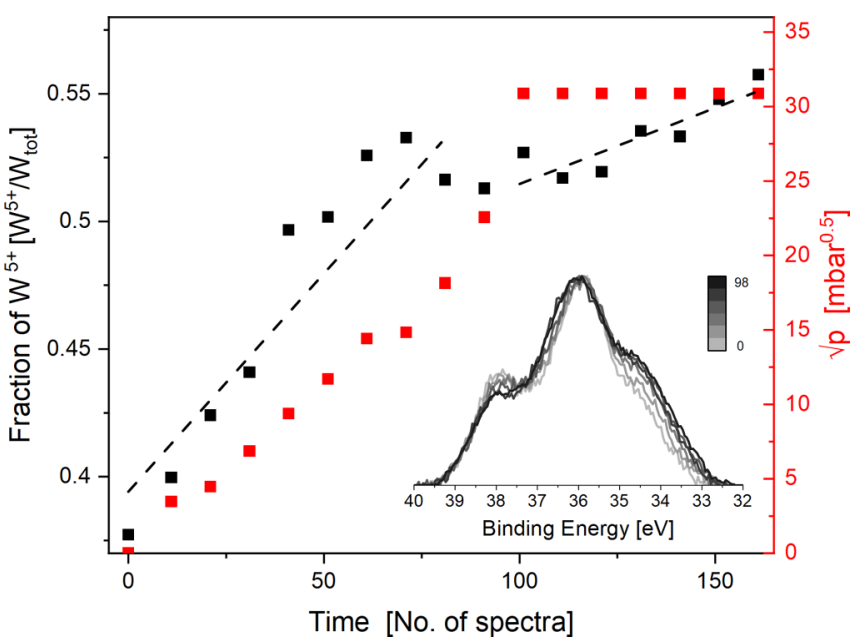

FIG. 3. The fraction of $\mathrm{W}^{5+}$ in the sample, as derived from peak fitting shown in the Appendix (Fig. 7), is shown in black, with the linear interpolation of the slope shown by the dashed gray lines during the increasing hydrogen pressure and the constant hydrogen pressure phases, shown in red. The grayscale spectra show the changes in the $\mathrm{W} 4 f$ spectra during the increasing hydrogen pressure phase. 


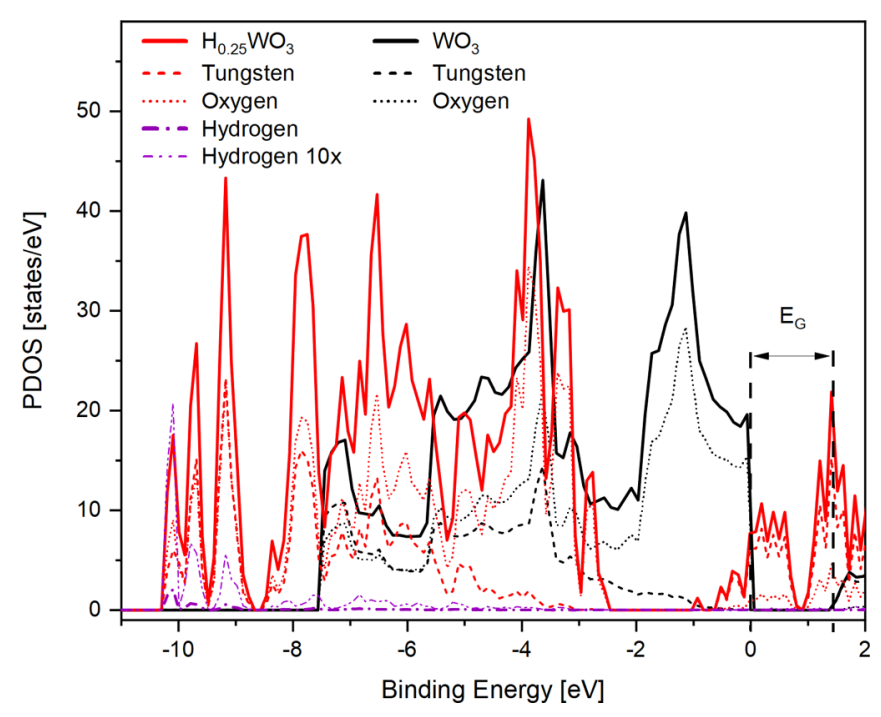

FIG. 4. Modeled partial DOS (PDOS) and total DOS of cubic $\mathrm{WO}_{3}$ (black line) and $\mathrm{H}_{x} \mathrm{WO}_{3}$ (red line) with hydrogen concentrations as small as $x=0.25 . E_{G}=1.3 \mathrm{eV}$ is the indirect band gap in $\mathrm{WO}_{3}$ derived from the onsets of valence and conduction bands. Full band structure calculations give the direct band gap of $2.3 \mathrm{eV}$ (not shown). Note the minimal contribution of hydrogen electrons (purple dash-dotted line) to the total density of states in $\mathrm{H}_{0.25} \mathrm{WO}_{3}$.

increasing hydrogen back pressure up to 1 bar and then kept under these conditions for a long period. The increase in the $\mathrm{W}^{5+}$ fraction shows two different slopes. With increasing the hydrogen back pressure, the amount of $\mathrm{W}^{5+}$ grows fast. When the hydrogen back pressure is high but constant, the growth of $\mathrm{W}^{5+}$ slows down markedly. The amount of $\mathrm{W}^{5+}$ purely from hydrogenation may then be the difference between the two dashed fitting curves, neglecting a mutual enhancement of the two effects. The hydrogen content in $\mathrm{H}_{x} \mathrm{WO}_{3}$ has been shown to be proportional to the square root of the applied hydrogen pressure $[18,44]$. With this relation, the amount of $\mathrm{W}^{5+}$ is proportional to $\sqrt{p_{\mathrm{H}_{2}}}$ and thus to the amount of hydrogen $x$ in $\mathrm{H}_{x} \mathrm{WO}_{3-\delta}$. An uncertainty is the number of oxygen vacancies $\delta$. The measured differences in the O-to- $\mathrm{W}$ elemental ratio between pre- and posthydrogenation are less than 3\% (Appendix, Fig. 8). This lies within the accuracy of the measurements. Within this limitation, there is no oxygen loss during hydrogenation.

The measurement of valence band spectra was performed in parallel to the $\mathrm{W} 4 f$ core levels, showing subtle changes with hydrogenation as well (Fig. 6). However, in contrast to the binding-energy shifts of core levels, which can be interpreted rather easily $[45,46]$, the $\mathrm{H} 1 s$ state is both core level as well as valence state, and thus not easily characterized. This challenge is tackled by an in-depth analysis of the valence band spectra with the help of DFT calculations.

DFT calculations were performed on simple cubic $\mathrm{WO}_{3}$, $\mathrm{H}_{0.25} \mathrm{WO}_{3}, \mathrm{H}_{0.75} \mathrm{WO}_{3}$, and $\mathrm{H}_{1} \mathrm{WO}_{3}$ to illustrate the effects of hydrogenation on the electronic structure. $\mathrm{WO}_{3}$ exhibits typical features of a semiconductor with a direct band gap of about $2.3 \mathrm{eV}$ and an indirect one of $1.3 \mathrm{eV}$ as derived from band structure calculations (see Fig. 4). As discussed in Sec. IID, the gap is underestimated (the experimental gap of monoclinic
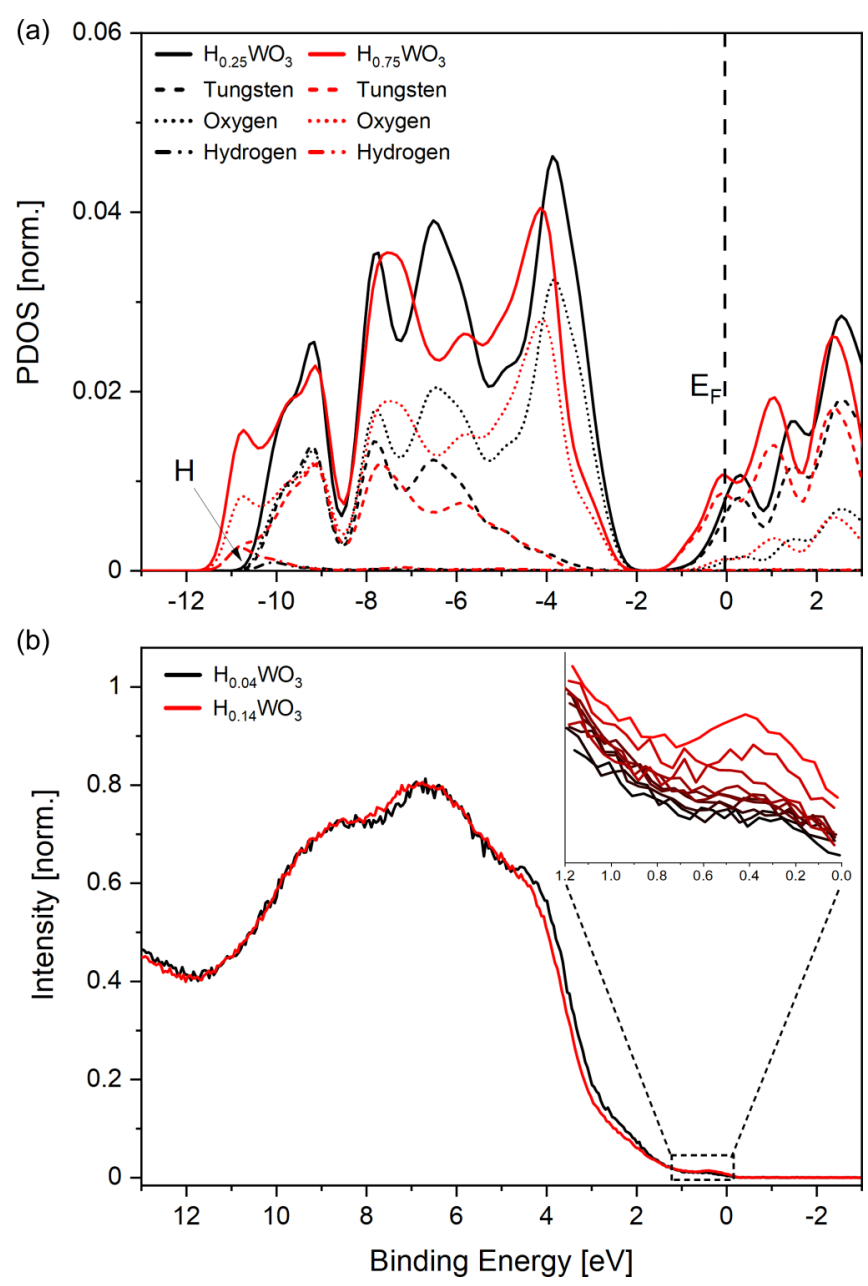

FIG. 5. (a) Calculated partial and total DOS of $\mathrm{H}_{0.25} \mathrm{WO}_{3}$ (black) and $\mathrm{H}_{0.75} \mathrm{WO}_{3}$ (red). (b) Measured photoemission spectra of minimum and maximum hydrogen content in black and red. The hydrogen contents as labeled are derived from the peak intensities of the peak evolving with hydrogen pressure as shown in the inset (see Fig. 6 and text for more information).

$\mathrm{WO}_{3}$ is $\left.3.2 \mathrm{eV}[13,37]\right)$ due to the assumption of a cubic lattice, which simplifies the calculation of hydrogen-intercalated $\mathrm{WO}_{3}$. Nevertheless, the overall electronic structure with the valence band edge dominated by oxygen orbitals is in perfect agreement with the literature. This is also in agreement with experimental observations: The optical absorption in crystalline $\mathrm{H}_{x} \mathrm{WO}_{3}$ is very similar to that in amorphous $\mathrm{H}_{x} \mathrm{WO}_{3}$ indicating that the crystallinity plays only a minor role in electronic structure changes upon hydrogenation [47].

The intercalation of hydrogen into $\mathrm{WO}_{3}$ was modeled by placing hydrogen in the center of the $\mathrm{WO}_{3}$ subunit and optimizing its position in the simple cubic structure by energy minimization. The minimum was found with hydrogen occupying a position near one oxygen site with a distance of 1.04 $\AA$ identical to the calculations of Hjelm et al. [8], and very similar to the bond length of an $\mathrm{OH}$ ion. Simultaneously, the lattice is slightly distorted (Fig. 2). The presence of hydrogen accompanied by the lattice distortion leads to two main changes in the electronic structure (see Fig. 4): The valence band broadens due to the bonding of oxygen with hydrogen 


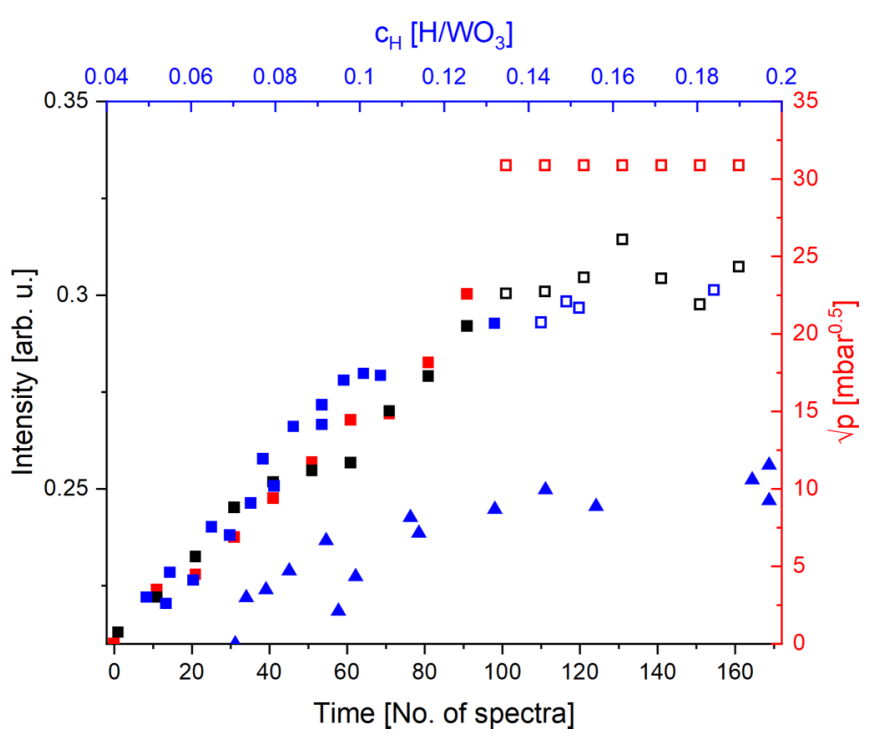

FIG. 6. The intensity of hydrogen-induced, conduction-bandlike tungsten states (black) are shown as a function of time and with hydrogen back pressure (red). Solid symbols indicate increasing hydrogen pressure, and open ones mean constant hydrogen back pressure. The bulk pressure-composition isotherm data on $\mathrm{Pt}: \mathrm{WO}_{3}$ (blue) at $423 \mathrm{~K}$ are plotted on the same pressure axis [18]. Squares represent absorption data, whereas triangles represent desorption data.

(bonding states around $-11 \mathrm{eV}$ ), and the conduction band minimum dominated by tungsten states is being occupied by electrons donated by the hydrogen and pushed below the Fermi level. It is expected that these changes lead to large increases in the electronic conductivity, which will be discussed in Sec. IV. However, the aim of this paper is to confirm these effects by photoelectron spectroscopy, as these states should be observable.

Figure 5 shows the calculated density of states (DOS) for $\mathrm{H}_{0.25} \mathrm{WO}_{3}$ and $\mathrm{H}_{0.75} \mathrm{WO}_{3}$ that have been broadened to account for the natural linewidth of the photoemission process and valence band spectra at low and high applied hydrogen back pressure. The effects of going from $\mathrm{H}_{0.25} \mathrm{WO}_{3}$ to $\mathrm{H}_{0.75} \mathrm{WO}_{3}$ (see Fig. 5) are less drastic compared with the difference between $\mathrm{WO}_{3}$ and $\mathrm{H}_{x} \mathrm{WO}_{3}$ (see Fig. 4). Most striking are the increasing hydrogen-oxygen bonding states and corresponding broadening of the oxygen states, which leads to a decrease in the DOS near $3 \mathrm{eV}$ binding energy. The second effect, most relevant to this paper, is the increase in tungsten states near $E_{F}$ with increasing hydrogen content. These two changes match the experimental spectra exceptionally well (Fig. 5). In particular, the calculations confirm the existence of hydrogen-induced states near $E_{F}$, which are associated with tungsten states.

The normalized peak area of these hydrogen-induced tungsten states at $0.4 \mathrm{eV}$ binding energy scales with the square root of the hydrogen back pressure (Fig. 6). It is important to note that it does not evolve any further with time at constant pressure, which excludes its origin induced by radiation damage and confirms that the measured state is in thermodynamic equilibrium with hydrogen gas. Since the number of hydrogen-induced tungsten states is directly re- lated to the applied hydrogen back pressure by employing our membrane approach, we plot it together with the bulk pressure-composition isotherm of $\mathrm{Pt}: \mathrm{WO}_{3}$ reported in the literature [18] using the common pressure axis. Using this calibration, we extract that the initial hydrogen content in our thin films is $\mathrm{H}_{0.04} \mathrm{WO}_{3}$ increasing to $\mathrm{H}_{0.14} \mathrm{WO}_{3}$ at 1000 mbar.

\section{DISCUSSION}

Already in the past, Hollinger et al. proposed that coloration in amorphous tungsten oxide films upon hydrogenation is connected to an increase in localized electronic states $\left(\mathrm{W}^{5+}\right)$, but they could not disentangle whether the origin of the effect was from oxygen defects caused by UV exposure or from hydrogen intercalation [48]. The optical absorption band corresponds to a peak near the Fermi level detected by photoelectron spectroscopy.

With this, our measurements confirm most of the literature data on electronic structure changes in $\mathrm{H}_{x} \mathrm{WO}_{3}$ or substoichiometric $\mathrm{WO}_{3-\delta}$, in particular, the evolution of the peak near the Fermi level $[14,23,41,43,48]$. However, with our experimental procedure we can disentangle the contributions from oxygen deficiency and hydrogen incorporation. The membrane photoemission measurements show that hydrogen interaction with $\mathrm{WO}_{3}$ leads to band-gap states without the loss of oxygen (Appendix, Fig. 8).

Attributing these states near the Fermi level to hydrogen is too short viewed. Even without sophisticated band structure calculations we can estimate the potential effect: The valence band of $\mathrm{H}_{x} \mathrm{WO}_{3}$ consists of $3 \times 6 \mathrm{O} 2 p$ states (we neglect here the small contribution of $\mathrm{W} 5 d$ ) and at most 0.2 hydrogens. In addition, we have to consider the photoionization cross section of electrons localized on hydrogen and oxygen of 0.02 and $1 \mathrm{Mb}$, respectively, at around $104 \mathrm{eV}$ [24]. The expected intensity ratio between hydrogen states and total valence states is $0.2 /(3 \times 6) \times 0.02 / 1=2.2 \times 10^{-4}$. It is thus unlikely that the photoelectrons near $E_{F}$ stem from hydrogen. Instead, the DFT modeling shows that hydrogenation of $\mathrm{WO}_{3}$ leads to the formation of $\mathrm{OH}$ with hydrogen states appearing at $-11 \mathrm{eV}$ and tungstenlike conduction band states near the Fermi level (Fig. 5).

Conductivity and optical experiments evidence that hydrogen-intercalated $\mathrm{WO}_{3}$ is not an electronic conductor at small hydrogen content in contrast to what is suggested by the calculated DOS (Fig. 4), which shows states at $E_{F}$. This discrepancy was already found in the past and explained by correlation models [49]. In the local picture, hydrogen intercalation gives

$$
\mathrm{W}^{6+} \mathrm{O}_{3}^{2-} \stackrel{\mathrm{H}}{\longrightarrow} \mathrm{W}^{5+}(\mathrm{OH})^{-} \mathrm{O}_{2}^{2-},
$$

with the electron from the hydrogen localized by the $\mathrm{OH}$ bond. With this, the nearby tungsten atom is different from the ones surrounded solely by oxygen atoms (Fig. 2). This local picture is still compatible with the band structure; however, dynamic properties depend on the correlation of the corresponding electrons. Such calculations are beyond the scope of this paper. We restrict ourselves to the following simplified picture explaining the observation that despite having electrons near the Fermi level (Fig. 5), $\mathrm{H}_{x} \mathrm{WO}_{3}$ does not exhibit metallic character (no Drude absorption [13]): The movement 
of electrons associated with the tungsten atom near the $\mathrm{OH}$ group $\left(\mathrm{W}^{5+}\right)$ is correlated with the hopping of the proton, which requires crystallographic rearrangement as the oxygen orientations around the tungsten atoms are slightly different (see Fig. 2).

Crandall and Faughnan [49] find a metal-insulator transition around hydrogen concentrations of approximately 0.3 , which is close to that predicted by percolation theory. At the percolation threshold, the hopping between the tungsten atoms will form a continuous chain throughout the crystal. Further evidence for the localization of these electrons was provided by optical absorption spectroscopy where amorphous $\mathrm{H}_{x} \mathrm{WO}_{3}$ exhibits an absorption maximum in the near-infrared range $[4,50]$. The optically induced jumping of the electron from one $\mathrm{W}$ site $\left(\mathrm{W}_{a}\right)$ to the other $\left(\mathrm{W}_{b}\right)$ is a special charge-transfer excitation, which is well described by the so-called polaron model [13]:

$$
\mathrm{W}_{\mathrm{a}}^{5+}(\mathrm{OH})+\mathrm{W}_{\mathrm{b}}^{6+} \mathrm{O} \stackrel{\hbar \omega}{\longrightarrow} \mathrm{W}_{\mathrm{a}}^{6+} \mathrm{O}+\mathrm{W}_{\mathrm{b}}^{5+}(\mathrm{OH}) .
$$

In contrast to Saenger et al. [13], who attribute the corresponding interaction of this electron to the surrounding distorted oxygen-deficient lattice, we propose the interaction to be between the proton and the electron on $\mathrm{W}^{5+}$ also leading to a distorted lattice. This so-called proton polaron has been proposed for describing the proton transport in a similar system, hydrated $\mathrm{BaCe}_{0.8} \mathrm{Y}_{0.2} \mathrm{O}_{3-\delta}$ [51]. The general underlying principle, the required crystalline rearrangements linked to charge transfer ("movement of distortions," Fig. 2; and energy dependence on hydrogen constellation, Appendix, Fig. 10), remains the same. The metal oxygen rearrangement proceeds through vibrations of the $\mathrm{WO}_{6}$ octahedra and $\mathrm{O}-\mathrm{H}$ vibrations. The mobility of protons may thus be in both cases a result of phonon-assisted jumps. The changes in the electronic structure by a proton-oxygen bond also explain the difference from changes induced by oxygen vacancies. Here, recent DFT calculations provide arguments that oxygen vacancies in $\mathrm{WO}_{3}$ introduce "free" carriers to the conduction band, because the corresponding electrons are delocalized over a large area [52]. In our case, the $\mathrm{OH}$ bond pins a similar electron brought into the system by hydrogen intercalation. From an electron spectroscopy point of view, this difference is indistinguishable, but the localized picture is in good agreement with other physical properties of $\mathrm{H}_{x} \mathrm{WO}_{3-\delta}$ (see discussion above).

Finally, tackling the difficulties of probing the electronic structure in $\mathrm{H}_{x} \mathrm{WO}_{3-\delta}$ by static electron spectroscopy by following dynamic changes in situ may be extended to ultrafast spectroscopy. With this, the degree of localization is directly accessible.

The main outcome of this study is the change in the electronic structure near the Fermi edge from oxygen-dominated orbitals to tungsten-dominated orbitals (see Fig. 4). This has drastic consequences for (electro)catalysis of oxygen-related reactions in addition to physical properties such as electron conductivity and optical properties as discussed above. $\mathrm{WO}_{3}$ is one of the oxides considered to be a suitable photoelectrochemical water-splitting catalyst [53]. In particular, it shows a high activity for water oxidation in the presence of a suitable electron acceptor [53,54]. Hydrogen treatment of $\mathrm{WO}_{3}$ can increase photocurrent significantly [54,55], which was interpreted as an increase in the number of oxygen vacancies, and thus defect states. With our findings, we cannot exclude the existence of oxygen defect states in parallel with proton polarons. However, the more pronounced effect of hydrogen treatment compared with vacuum annealing $[54,55]$ suggests that generally, hydrogen-related states are the main origin of the effect.

Similarly, the hydrogen-induced electronic structure near the Fermi edge explains the rather small effect on superconductivity in tungsten oxides, if compared with metal doping $[56,57]$. If hydrogen vibrations with $\mathrm{H}$ PDOS at $E_{F}$ contributed to the superconductivity, a strong positive effect may be expected. However, the superconductivity in bulk $\mathrm{WO}_{3}$ is currently understood to originate from a weak-coupling
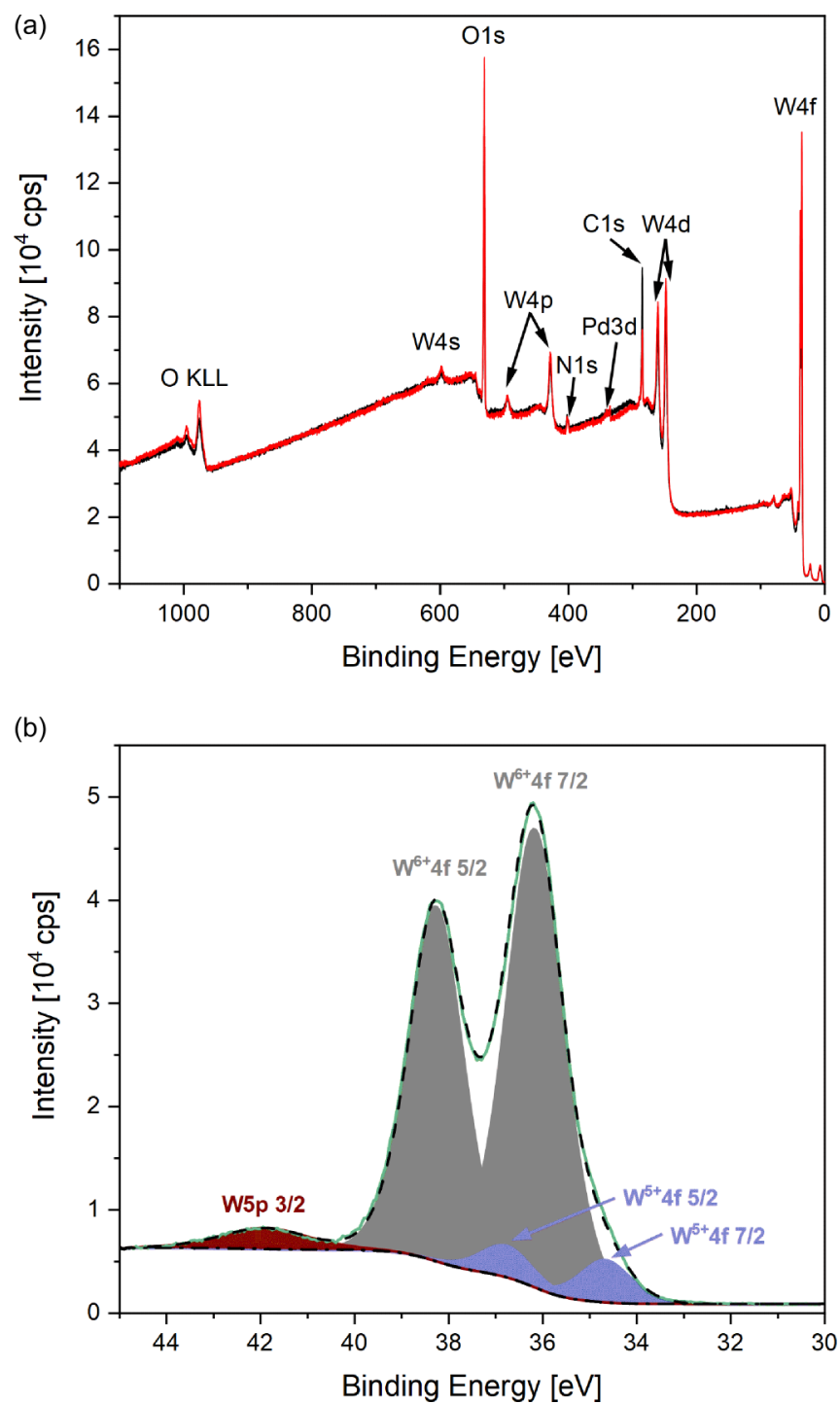

FIG. 7. (a) The pre- and posthydrogenation XPS survey spectra are shown in black and red, respectively. (b) The W $5 p_{3 / 2}$ (brown) and $\mathrm{W} 4 f$ (gray and violet) core levels of the electrodeposited $\mathrm{WO}_{3}$ as prepared, as described in Sec. II A, measured by Al K $\alpha$ XPS. The spectra are fitted with two pairs of doublets giving the $\mathrm{W}^{6+}$ and $\mathrm{W}^{5+}$ fractions, respectively. The peak fitting indicates the sample being almost completely oxidized, with a small fraction of $\mathrm{W}^{5+}$ states present $(\simeq 7.7 \%)$. 
state sustained by soft vibrational modes of the $\mathrm{WO}_{6}$ octahedra [57], and the formation of $\mathrm{OH}$ instead of the creation of oxygen vacancies upon hydrogenation is in line with this argumentation.

\section{CONCLUSIONS}

We measured the palladium-assisted hydrogenation of tungsten trioxide by operando membrane XPS at hydrogen pressures up to 1 bar. The combination of the membrane XPS with synchrotron radiation allows us to measure both core levels and the valence band simultaneously up to high hydrogen pressures minimizing the effect of initial preparation parameters, beam damage, and exposure to the reducing UHV environment that would occur with consecutive XPS or ultraviolet photoelectron spectroscopy (UPS) measurements. Analysis of the tungsten $4 f$ core levels shows an increase in $\mathrm{W}^{5+}$ with increasing hydrogen pressure. At the same time a state appears
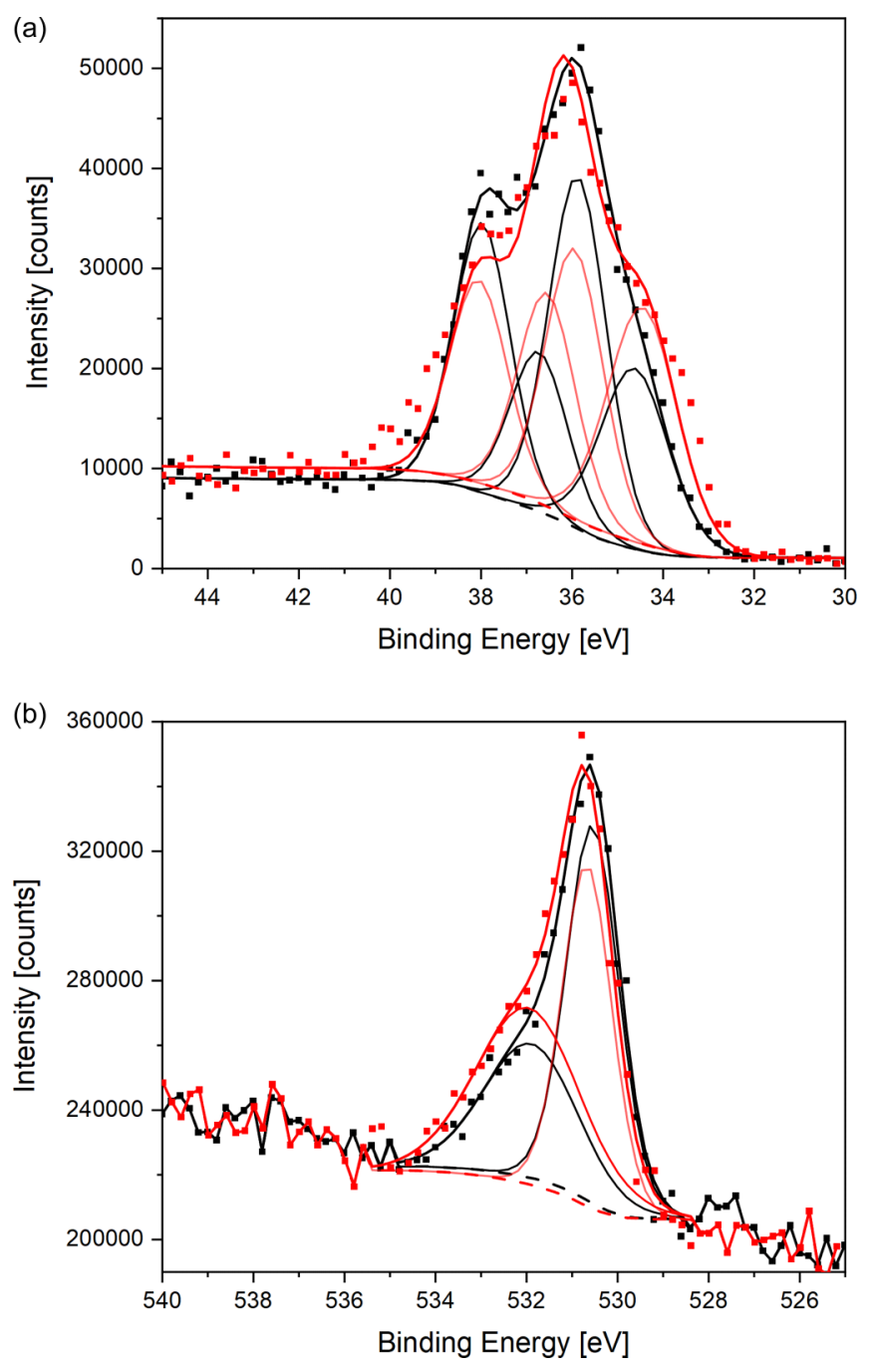

FIG. 8. W $4 f$ (a) and O $1 s$ (b) core-level spectra recorded using $700 \mathrm{eV}$ photon energy. The spectra were recorded in situ before and after exposure to hydrogen (black and red, respectively). The $\mathrm{W}^{5+}$ fraction increases from 37.6 to $51.2 \%$ and the oxygen defect fraction from 38.8 to $52.0 \%$ upon hydrogenation. The intensity ratio of $\mathrm{W}$ to $\mathrm{O}$ remains nearly constant with $I\left(\mathrm{O}_{\text {latt }}\right) / I(\mathrm{~W})=1.06 \rightarrow 1.03$. close to the Fermi level that is connected to the hydrogen content in the compound. Although induced by hydrogen, the corresponding electrons are allocated to tungsten $d$ states. Using previous reference measurements, it is possible to create a pressure-composition isotherm from the spectroscopy data. The photoemission measurements together with DFT calculations corroborate that the coloration of the films by hydrogen can be explained by a proton polaron model.

\section{ACKNOWLEDGMENTS}

This work was partly supported by the UZH-UFSP program LightChEC. Financial support from the Swiss National Science Foundation (Grant No. 172662 and Requip Grant No. 182987) is greatly acknowledged. We thank Dr. C. Puglia (Uppsala University, Sweden) and the Carl Tygger Foundation for the availability of VG Scienta's SES-200 photoelectron analyzer at the GasPhase beamline.

\section{APPENDIX}

\section{Experimental details of the photoemission experiments}

Table I shows the acquisition parameters used for XPS and synchrotron photoelectron spectroscopy.

Figures 7 and 8 compare the survey and W $4 f$ core-level spectra as measured ex situ (XPS) and in situ (synchrotron photoemission) before and after hydrogenation. In addition, the deconvolution of the spectra to derive the $\mathrm{W}^{6+}$ and $\mathrm{W}^{5+}$ fractions is shown for one archetypal example. From the intensity ratio of $\mathrm{O} 1 s$ to $\mathrm{W} 4 f$, one can estimate that the oxygen loss is below $3 \%$.

The rate-limiting step of the permeation of hydrogen through the membrane can be derived from the pressure dependence on both sides of the membrane:

$$
p_{\mathrm{UHV}} \propto p_{\mathrm{appl}}^{r}
$$

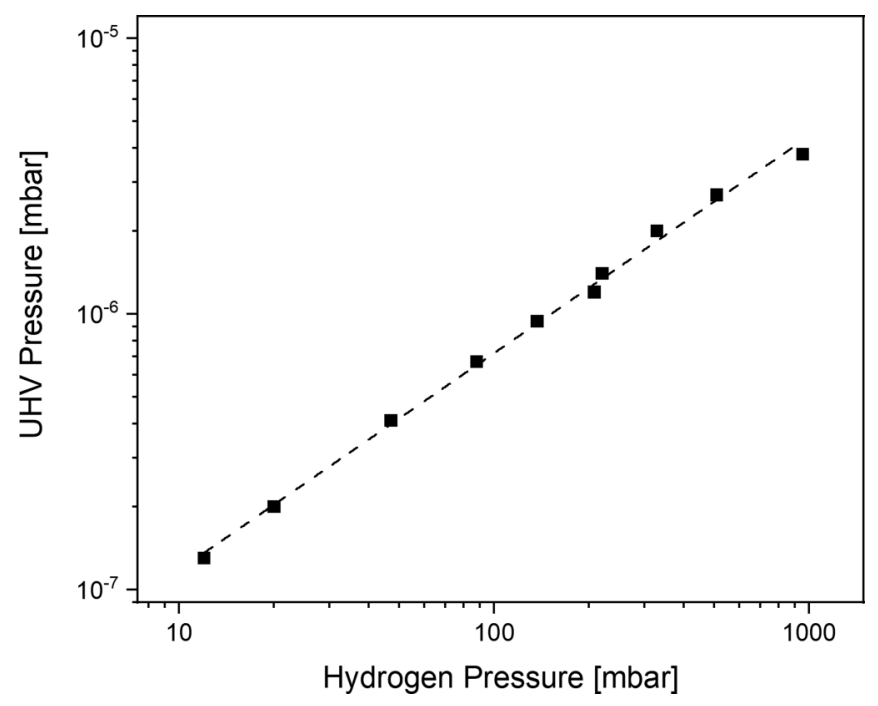

FIG. 9. Double-log plot of the measured hydrogen pressure in the vacuum chamber as a function of the applied feed pressure. A fit yields a slope of 0.79 . 

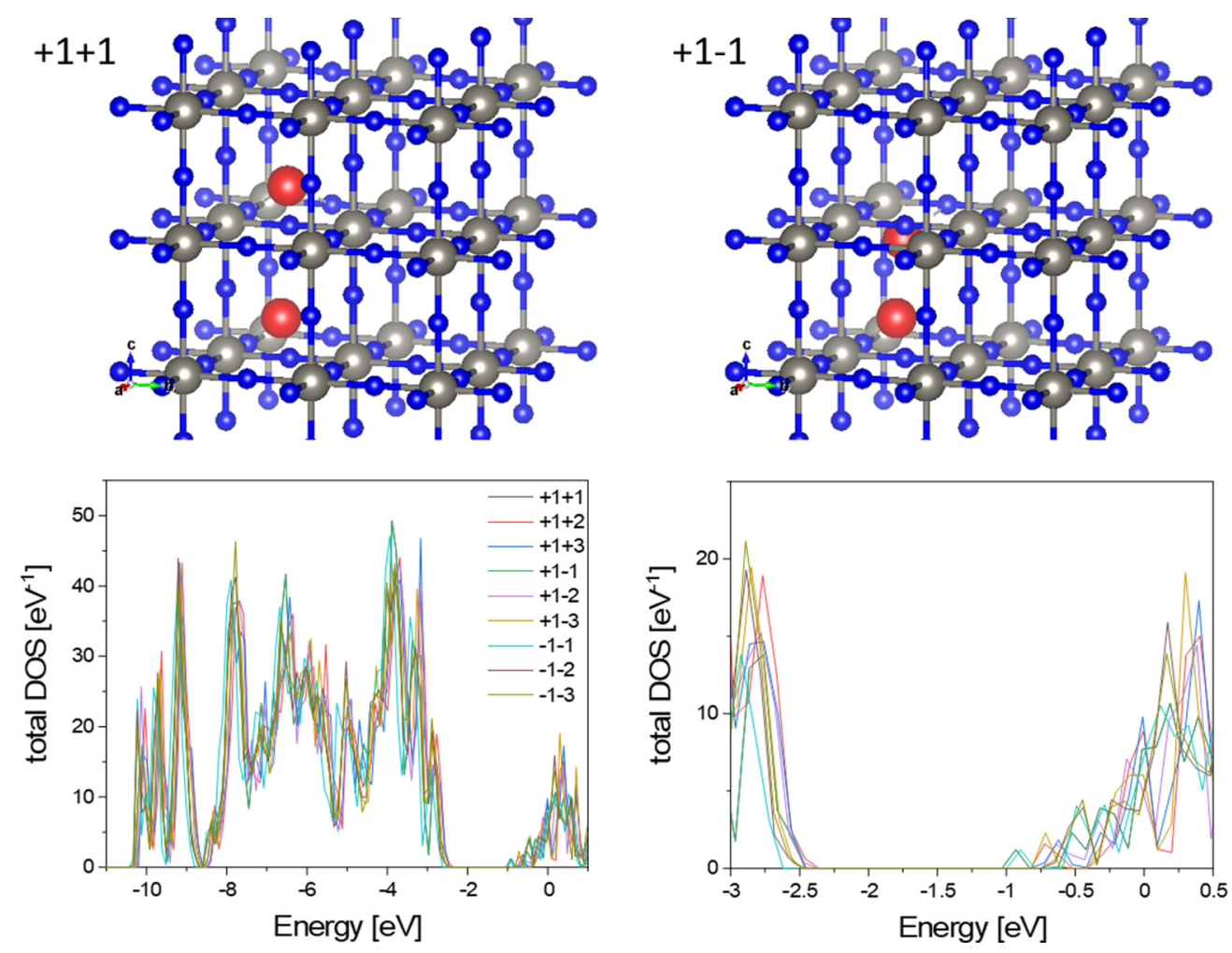

FIG. 10. Top: As an example, two different hydrogen constellations in $\mathrm{H}_{0.25} \mathrm{WO}_{3}$, denoted as $+1+1$ and $+1-1$, before relaxation. Bottom: DFT of nine different constellations. The right graph is an enlargement around the band gap.

For ideal cases, surface-limited permeation gives an exponent $r=1$, and bulk diffusion gives $r=\frac{1}{2}[19,20]$. A fit to the double-log plot of the measured hydrogen pressure in the vacuum chamber as a function of the applied feed pressure yields an exponent of 0.79 (Fig. 9), indicative of surface-limited kinetics.

\section{Hydrogen constellations used for DFT calculations}

Substoichiometric concentrations $(x<1)$ in $\mathrm{H}_{x} \mathrm{WO}_{3}$ are difficult to model. For $x=0.25$, the unit cell of $\mathrm{H}_{0.25} \mathrm{WO}_{3}$ is extended to a $2 \times 2 \times 2$ supercell of the simple unit cell of $\mathrm{H}_{1} \mathrm{WO}_{3}$ based on one $\mathrm{H}$ in the $\mathrm{WO}_{3}$ cell (Fig. 10). Not all of the now possible $\mathrm{H}$ positions are occupied to reflect the substoichiometry giving additional freedom as to where to place these hydrogen atoms relative to each other. We modeled nine archetypal constellations, where the hydrogen atoms are placed in the subcells along the 100, 110, and 111 directions and with different spin constellations. The corresponding energy shifts are of the order of $0.3 \mathrm{eV}$. This difference is neglected within the interpretation of the photoelectron spectra; however, the nonzero energy difference will contribute to the energy barrier for hydrogen motion in $\mathrm{H}_{x} \mathrm{WO}_{3}$.
[1] J. Völkl and H. Wipf, Diffusion of hydrogen in metals, Hyperfine Interact. 8, 631 (1981).

[2] F. Wöhler, Ueber das Wolfram, Ann. Phys. (Berlin) 78, 345 (1824).

[3] E. O. Brimm, J. C. Brantley, J. H. Lorenz, and M. H. Jellinek, Sodium and potassium tungsten bronzes, J. Am. Chem. Soc. 73, 5427 (1951).

[4] S. K. Deb, Optical and photoelectric properties and colour centres in thin films of tungsten oxide, Philos. Mag. 27, 801 (1973).

[5] C. G. Granqvist, Electrochromic tungsten oxide films: Review of progress 1993-1998, Sol. Energy Mater. Sol. Cells 60, 201 (2000).

[6] H. Höchst, R. Bringans, H. Shanks, and P. Steiner, Failure of the rigid band model in $\mathrm{Na}_{x} \mathrm{WO}_{3}$ : An XPS study, Solid State Commun. 37, 41 (1981).
[7] S. Raj, T. Sato, S. Souma, T. Takahashi, D. D. Sarma, and P. Mahadevan, Metal-insulator transition of $\mathrm{Na}_{x} \mathrm{WO}_{3}$ studied by angle-resolved photoemission spectroscopy, Mod. Phys. Lett. B 23, 2819 (2009).

[8] A. Hjelm, C. G. Granqvist, and J. M. Wills, Electronic structure and optical properties of $\mathrm{WO}_{3}$, $\mathrm{LiWO}_{3}, \mathrm{NaWO}_{3}$, and $\mathrm{HWO}_{3}$, Phys. Rev. B 54, 2436 (1996).

[9] A. L. Larsson, B. E. Sernelius, and G. A. Niklasson, Optical absorption of Li-intercalated polycrystalline tungsten oxide films: Comparison to large polaron theory, Solid State Ionics 165, 35 (2003).

[10] C. G. Granqvist, Bulk crystalline tungsten oxide, in Handbook of Inorganic Electrochromic Materials (Elsevier, New York, 1995), Chap. 2, p. 633. 
[11] H. Höchst, R. D. Bringans, and H. R. Shanks, Electronic structure of $\mathrm{Na}_{x} \mathrm{WO}_{3}$ : A photoemission study covering the entire concentration range, Phys. Rev. B 26, 1702 (1982).

[12] X. Leng, J. Pereiro, J. Strle, G. Dubuis, A. T. Bollinger, A. Gozar, J. Wu, N. Litombe, C. Panagopoulos, D. Pavuna, and I. Božović, Insulator to metal transition in $\mathrm{WO}_{3}$ induced by electrolyte gating, npj Quantum Mater. 2, 35 (2017).

[13] M. F. Saenger, T. Höing, B. W. Robertson, R. B. Billa, T. Hofmann, E. Schubert, and M. Schubert, Polaron and phonon properties in proton intercalated amorphous tungsten oxide thin films, Phys. Rev. B 78, 245205 (2008).

[14] M. B. Johansson, P. T. Kristiansen, L. Duda, G. A. Niklasson, and L. Österlund, Band gap states in nanocrystalline $\mathrm{WO}_{3}$ thin films studied by soft x-ray spectroscopy and optical spectrophotometry, J. Phys.: Condens. Matter 28, 475802 (2016).

[15] P. G. Dickens and R. J. Hurditch, X-ray and neutron diffraction studies of a tetragonal hydrogen bronze $\mathrm{H}_{x} \mathrm{WO}_{3}$, Nature (London) 215, 1266 (1967).

[16] P. Wiseman and P. Dickens, The crystal structure of cubic hydrogen tungsten bronze, J. Solid State Chem. 6, 374 (1973).

[17] G. A. de Wijs and R. A. de Groot, Structure and electronic properties of amorphous $\mathrm{WO}_{3}$, Phys. Rev. B 60, 16463 (1999).

[18] A. R. Berzins and P. A. Sermon, Reversible isothermal sorption of hydrogen by tungsten trioxide in presence of platinum, Nature (London) 303, 506 (1983).

[19] R. Delmelle, B. Probst, R. Alberto, A. Züttel, D. Bleiner, and A. Borgschulte, Closing the pressure gap in X-ray photoelectron spectroscopy by membrane hydrogenation, Rev. Sci. Instrum. 86, 053104 (2015).

[20] O. Sambalova and A. Borgschulte, Membrane concept for environmental surface science, J. Alloys Compd. 742, 518 (2018).

[21] R. Blyth, R. Delaunay, M. Zitnik, J. Krempasky, R. Krempaska, J. Slezak, K. Prince, R. Richter, M. Vondracek, R. Camilloni, L. Avaldi, M. Coreno, G. Stefani, C. Furlani, M. de Simone, S. Stranges, and M.-Y. Adam, The high resolution gas phase photoemission beamline, Elettra, J. Electron Spectrosc. Relat. Phenom. 101-103, 959 (1999).

[22] C. Grazioli, Photoionization experiments in the study of energy transfer in nanostructured materials and their precursors, Ph.D. thesis, Università degli Studi di Trieste, 2017.

[23] O. Bouvard, A. Krammer, and A. Schüler, In situ core-level and valence-band photoelectron spectroscopy of reactively sputtered tungsten oxide films, Surf. Interface Anal. 48, 660 (2016).

[24] J. J. Yeh and I. Lindau, Atomic subshell photoionization cross sections and asymmetry parameter: $1<\mathrm{Z}<103$, At. Data Nucl. Data Tables 32, 1 (1985).

[25] G. Kresse and J. Hafner, Ab initio molecular dynamics for liquid metals, Phys. Rev. B 47, 558 (1993).

[26] G. Kresse and J. Hafner, Ab initio molecular-dynamics simulation of the liquid-metal-amorphous-semiconductor transition in germanium, Phys. Rev. B 49, 14251 (1994).

[27] G. Kresse and J. Furthmüller, Efficiency of ab-initio total energy calculations for metals and semiconductors using a plane-wave basis set, Comput. Mater. Sci. 6, 15 (1996).

[28] G. Kresse and J. Furthmüller, Efficient iterative schemes for $a b$ initio total-energy calculations using a plane-wave basis set, Phys. Rev. B 54, 11169 (1996).

[29] P. E. Blochl, Projector augmented-wave method, Phys. Rev. B 50, 17953 (1994).
[30] G. Kresse and D. Joubert, From ultrasoft pseudopotentials to the projector augmented-wave method, Phys. Rev. B 59, 1758 (1999).

[31] J. P. Perdew, K. Burke, and M. Ernzerhof, Generalized Gradient Approximation Made Simple, Phys. Rev. Lett. 77, 3865 (1996).

[32] J. Heyd, G. E. Scuseria, and M. Ernzerhof, Hybrid functionals based on a screened Coulomb potential, J. Chem. Phys. 118, 8207 (2003).

[33] A. V. Krukau, O. A. Vydrov, A. F. Izmaylov, and G. E. Scuseria, Influence of the exchange screening parameter on the performance of screened hybrid functionals, J. Chem. Phys. 125, 224106 (2006).

[34] E. Lassner and W.-D. Schubert, Tungsten Properties, Chemistry, Technology of the Element, Alloys, and Chemical Compounds, 1st ed. (Springer, New York, 1999).

[35] H. A. Wriedt, The O-W (oxygen-tungsten) system, Bull. Alloy Phase Diagrams 10, 368 (1989).

[36] P. P. González-Borrero, F. Sato, A. N. Medina, M. L. Baesso, A. C. Bento, G. Baldissera, C. Persson, G. A. Niklasson, C. G. Granqvist, and A. Ferreira da Silva, Optical band-gap determination of nanostructured $\mathrm{WO}_{3}$ film, Appl. Phys. Lett. 96, 061909 (2010).

[37] M. Green and Z. Hussain, Optical properties of dilute hydrogen tungsten bronze thin films, J. Appl. Phys. (Melville, NY) 69, 7788 (1991).

[38] K. Xiong, J. Robertson, and S. J. Clark, Behavior of hydrogen in wide band gap oxides, J. Appl. Phys. (Melville, NY) 102, 083710 (2007).

[39] F. Wang, C. Di Valentin, and G. Pacchioni, Electronic and structural properties of $\mathrm{WO}_{3}$ : A systematic hybrid DFT study, J. Phys. Chem. C 115, 8345 (2011).

[40] F. Cora, A. Patel, N. M. Harrison, R. Dovesi, and C. R. A. Catlow, An ab initio Hartree-Fock study of the cubic and tetragonal phases of bulk tungsten trioxide, J. Am. Chem. Soc. 118 12174 (1996).

[41] B. A. De Angelis and M. Schiavello, X-ray photoelectron spectroscopy study of nonstoichiometric tungsten oxides, J. Solid State Chem. 21, 67 (1977).

[42] D. Barreca, G. Carta, A. Gasparotto, G. Rossetto, E. Tondello, and P. Zanella, A study of nanophase tungsten oxides thin films by XPS, Surf. Sci. Spectra 8, 258 (2001).

[43] F. Bussolotti, L. Lozzi, M. Passacantando, S. La Rosa, S. Santucci, and L. Ottaviano, Surface electronic properties of polycrystalline $\mathrm{WO}_{3}$ thin films: A study by core level and valence band photoemission, Surf. Sci. 538, 113 (2003).

[44] J. J. Fripiat and X. Lin, Hydrogen intercalation within transition metal oxides: Entropy, enthalpy, and charge transfer, J. Phys. Chem. 96, 1437 (1992).

[45] S. J. Kerber, J. J. Bruckner, K. Wozniak, S. Seal, S. Hardcastle, and T. L. Barr, The nature of hydrogen in X-ray photoelectron spectroscopy: General patterns from hydroxides to hydrogen bonding, J. Vac. Sci. Technol., A 14, 1314 (1996).

[46] J. L. Bourque, M. C. Biesinger, and K. M. Baines, Chemical state determination of molecular gallium compounds using XPS, Dalton Trans. 45, 7678 (2016).

[47] O. F. Schirmer and E. Salje, Conduction bipolarons in lowtemperature crystalline $\mathrm{WO}_{3-x}$, J. Phys. C: Solid State Phys. 13, L1067 (1980). 
[48] G. Hollinger, T. Minh Duc, and A. Deneuville, Charge Transfer in Amorphous Colored $\mathrm{WO}_{3}$ Films Observed by XRay Photoelectron Spectroscopy, Phys. Rev. Lett. 37, 1564 (1976).

[49] R. S. Crandall and B. W. Faughnan, Electronic Transport in Amorphous $\mathrm{H}_{x} \mathrm{WO}_{3}$, Phys. Rev. Lett. 39, 232 (1977).

[50] V. Wittwer, O. F. Schirmer, and P. Schlotter, Disorder dependence and optical detection of the Anderson transition in amorphous $\mathrm{H}_{x} \mathrm{WO}_{3}$ bronzes, Solid State Commun. 25, 977 (1978).

[51] A. Braun and Q. Chen, Experimental neutron scattering evidence for proton polaron in hydrated metal oxide proton conductors, Nat. Commun. 8, 15830 (2017).

[52] W. Wang, A. Janotti, and C. G. Van De Walle, Role of oxygen vacancies in crystalline $\mathrm{WO}_{3}$, J. Mater. Chem. C 4, 6641 (2016).
[53] S. S. Kalanur, L. T. Duy, and H. Seo, Recent progress in photoelectrochemical water splitting activity $\mathrm{WO}_{3}$ photoanodes, Top. Catal. 61, 1043 (2018).

[54] J. Yan, T. Wang, G. Wu, W. Dai, N. Guan, L. Li, and J. Gong, Tungsten oxide single crystal nanosheets for enhanced multichannel solar light harvesting, Adv. Mater. (Weinheim) 27, 1580 (2015).

[55] T. Singh, R. Müller, J. Singh, and S. Mathur, Tailoring surface states in $\mathrm{WO}_{3}$ photoanodes for efficient photoelectrochemical water splitting, Appl. Surf. Sci. 347, 448 (2015).

[56] S. Reich, G. Leitus, R. Popovitz-Biro, A. Goldbourt, and S. Vega, A possible $2 \mathrm{D} \mathrm{H}_{x} \mathrm{WO}_{3}$ superconductor with a $T_{c}$ of 120 K, J. Supercond. Novel Magn. 22, 343 (2009).

[57] C. Pellegrini, H. Glawe, and A. Sanna, Density functional theory of superconductivity in doped tungsten oxides, Phys. Rev. Materials 3, 064804 (2019). 